Received: 29.08 .2019

Revised: 18.10 .2019

Accepted: 25.10 .2019

DOI: $10.17804 / 2410-9908.2019 .5 .006-022$

\title{
COMPARISON OF THE YIELD FUNCTIONS FOR MICROPOLAR MEDIA USING A CYLINDRICAL SAMPLE MODEL
}

\author{
K. P. Frolova ${ }^{* a}$, E. N. Vilchevskaya ${ }^{\text {b) }}$ \\ Institute for Problems in Mechanical Engineering RAS \\ 61 Bolshoy Ave., V.O., St. Petersburg, Russian Federation \\ a) (iD https://orcid.org/0000-0003-0376-4463 kspfrolova@ gmail.com; \\ b) iD https://orcid.org/0000-0002-5173-3218 vilchevska@ gmail.com \\ *Corresponding author: E-mail: kspfrolova@gmail.com \\ Address for correspondence: V.O., Bolshoy pr., 61, St. Petersburg, 199178, Russian Federation \\ Tel.: +7 98180337 56; fax: +781232147 71
}

The article discusses different yield functions extended to the case of micropolar media in order to investigate the effect of local plasticity in metals. The paper is concerned with the inhomogeneous stress-strain state obtained within the frame of the micropolar continuum approach for a cylindrical specimen under distributed couple stress on its lateral surface. The case corresponding to specimen tension is considered. Plastic strains prove to arise near the border of the specimen, while the material continues to behave elastically. According to the results, it is necessary to take into account couple stresses to clarify what happens in the area near the border when the size of this area is comparable to the size of the structure inhomogeneity.

Keywords: yield function for a micropolar medium, size effects, pseudo-Cosserat continuum, couple stress.

\section{References}

1. Fizicheskaya mezomekhanika i kompyuternoe konstruirovaniye [Panin V.E., ed., Physical Mesomechanics and the Computer Design of Materials]. Novosibirsk, Nauka Publ., 1995. (In Russian).

2. Panin V.E., Grinyaev Yu.V., Psakhye S.G. Two decades of developments in physical mesomechanics: achievements, problems and prospects. Fizicheskaya Mezomekhanika, 2004, vol. 7, no. 4, pp. 93-113. (In Russian).

3. Tyumentsev A.N., Korotayev A.D., Pinzhin Yu.P. Highly Defective Structural States, Fields of Local Internal Stresses and Cooperative Mesoscopic Mechanisms of Crystal Deformation and Reorientation in Nanostructured Metal Materials. Phys. Mesomech., 2004, vol. 7, nos. 3-4, pp. 31-46.

4. Panin V.E., Panin A.V. Effect of the Surface Layer in a Solid under. Fizicheskaya Mezomekhanika, 2005, vol. 8, nos. 5-6, pp. 7-14. (In Russian).

5. Kuznetsov P.V. and Panin V.E., Direct Observation of Flows of Defects and of nm-Range Localization of Deformation on Duralumin Surface with the Aid of Scanning Tunnel and Atom Force Microscopes. Phys. Mesomech., 2000, vol. 3, no. 2, pp. 85-91.

6. Panin V.E., Yelsukova T.F., Popkova Yu.F., Pochivalov Yu.I. Role of curvature of the surface layer of titanium polycrystals in generation and development of fatigue fracture. Problems of Atomic Science and Technology, 2015, vol. 96, no. 2, pp. 148-153. (In Russian).

7. $\quad$ Panin V.E., Egorushkin V.E., Panin A.V., Moiseenko D.D. On the nature of plastic strain localization in solids. Technical Physics, 2017, vol. 52, iss. 8, pp 1024-1030. DOI: 10.1134/S1063784207080105. 
8. Eremeyev V.A., Lebedev L.P., Altenbach H. Foundations of Micropolar Mechanics, Springer Science \& Business Media, 2012.

9. Eringen A.C. Theory of micropolar elasticity. In: Microcontinuum Field Theories, Springer, New York, NY, 1999, pp. 101-248. DOI: 10.1007/978-1-4612-0555-5_5.

10. Mindlin R.D. Micro-structure in linear elasticity. Archive for Rational Mechanics and Analysis, 1964, vol. 16, no. 1, pp. 51-78. DOI: 10.1007/BF00248490.

11. Mindlin R.D., Tiersten H.F. Effects of couple-stresses in linear elasticity. Archive for Rational Mechanics and analysis, 1962, vol. 11, no. 1, pp. 415-448.

12. Kozlov E.V., Koneva N.A., and Zhdanov A.N. Structure and Strain Resistance of FCC U1trafine-Grained Metals and Alloys. Fizicheskaya Mezomekhanika, 2004, vol. 7, no. 4, pp. 93-113. (In Russian).

13. Forest S., Sievert R. Elastoviscoplastic constitutive frameworks for generalized continua. Acta Mechanica, 2003, vol. 160, nos. 1-2, pp. 71-111. DOI: 10.1007/s00707-002-0975-0.

14. Smolin I.Yu. The use of the micropolar models for the description of the plastic deformations at the mesoscale level. Vestnik Permskogo natsyonalnogo issledovatelskogo politekhnicheskogo universiteta: Mekhanika, 2006, no. 14, pp. 189-205. (In Russian).

15. Lippmann H. Eine Cosserat-Theorie des plastischen Fließens. Acta Mechanica, 1969, vol. 8, nos. 3-4, pp. 255-284. DOI: 10.1007/BF01182264.

16. Sawczuk A. On the yielding of Cosserat continua. Archives of Mechanics, 1967, vol. 19, no. 3, pp. 471-480.

17. Besdo D. Ein beitrag zur nichtlinearen theorie des Cosserat-kontinuums. Acta Mechanica, 1974, vol. 20, nos. 1-2, pp. 105-131. DOI: 10.1007/BF01374965.

18. Mühlhaus H.B., Vardoulakis I. The thickness of shear bands in granular materials. Geotechnique, 1987, vol. 37, no. 3, pp. 271-283. DOI: 10.1680/geot.1987.37.3.271.

19. De Borst R. Simulation of strain localization: a reappraisal of the Cosserat continuum. Engineering Computations, 1991, vol. 8, no. 4, pp. 317-332. DOI: 10.1108/eb023842.

20. De Borst R. A generalisation of J2-flow theory for polar continua. Computer Methods in Applied Mechanics and Engineering, 1993, vol. 103, no. 3, pp. 347-362. DOI: 10.1016/00457825(93)90127-J.

21. Lippmann H. Cosserat Plasticity and Plastic Spin. Appl. Mech. Rev., 1995, vol. 48, no. 11. DOI: $10.1115 / 1.3005091$.

22. Altenbach H., Eremeyev V.A. Strain rate tensors and constitutive equations of inelastic micropolar materials. International Journal of Plasticity, 2014, vol. 63, pp. 3-17. DOI: 10.1016/j.ijplas.2014.05.009.

23. Dietsche A., Steinmann P., Willam K. Micropolar elastoplasticity and its role in localization. International Journal of Plasticity, 1993, vol. 9, no. 7, pp. 813-831. DOI: 10.1016/07496419(93)90053-S.

24. Steinmann P. A micropolar theory of finite deformation and finite rotation multiplicative elastoplasticity. International Journal of Solids and Structures, 1994, vol. 31, no. 8, pp. 1063-1084. DOI: 10.1016/0020-7683(94)90164-3.

25. Kachanov L.M. Osnovy teorii plastichnosti [Fundamentals of Plasticity Theory]. Moscow, Nauka Publ., 1969, 420 p. (In Russian).

26. Mase G.E. Theory and Problems of Continuum, McGraw-Hill Book Company, New York, 1970.

27. Vardoulakis I. Shear-banding and liquefaction in granular materials on the basis of a Cosserat continuum theory. Ingenieur-Archiv., 1989, vol. 59, no. 2, pp. 106-113. DOI: $10.1007 / \mathrm{BF} 00538364$.

28. Salehi S.H., Salehi M. Numerical investigation of nanoindentation size effect using micropolar theory. Acta Mechanica, 2014, vol. 225, no. 12, pp. 3365-3376. DOI: 10.1007/s00707014-1116-2.

29. Neuber H. Über Probleme der Spannungskonzentration im Cosserat-Kцrper. Acta Mechanica, 1966, vol. 2, no. 1, pp. 48-69. DOI: 10.1007/BF01176729. 
30. Groen A.E., Schellekens J.C.J., De Borst R. Three-dimensional finite element studies of failure in soil bodies using a Cosserat continuum. In: Computer Methods and Advances in Geomechanics: Proceedings of the 8th International Conference on Computer Methods and Advances in Geomechanics, Morgantown, West Virginia, USA, 22-28 May 1994, Balkema, 1994, pp. 581-586.

31. Frolova K., Vilchevskaya E., Polyanskiy V., Alekseeva E. Modelling of a Hydrogen Saturated Layer Within the Micropolar Approach. In: New Achievements in Continuum Mechanics and Thermodynamics, Springer, Cham, 2019, pp. 117-128. DOI: 10.1007/978-3-030-13307-8_9.

32. Adomeit G. Determination of elastic constants of a structured material. In: Mechanics of Generalized Continua, Springer, Berlin, Heidelberg, 1968, pp. 80-82. DOI: 10.1007/978-3-66230257-6_8.

33. Lakes R.S. Size effects and micromechanics of a porous solid. Journal of Materials Science, 1983, vol. 18, no. 9, pp. 2572-2580. DOI: 10.1007/BF00547573.

34. Yang J.F.C., Lakes R.S. Transient study of couple stress effects in compact bone: torsion. Journal of Biomechanical Engineering, 1981, vol. 103, no. 4, pp. 275-279.

35. Alekseeva E., Belyaev A., Zegzhda A., Polyanskiy A., Polyanskiy V., Frolova K., Yakovlev Y. Boundary layer influence on the distribution of hydrogen concentrations during hydrogen-induced cracking test of steels. Diagnostics, Resource and Mechanics of materials and structures, 2018, iss. 3, pp. 43-57. DOI: 10.17804/2410-9908.2018.3.043-057.

36. Belyaev A.K., Polyanskiy V.A., Yakovlev Y.A., Mansyrev D.E., Polyanskiy A.M. Surface effect of the waves of plastic deformation and hydrogen distribution in metals. In: Days on Diffraction: IEEE, 2017, pp. 45-50. DOI: 10.1109/DD.2017.8167993.

37. Polyanskiy V.A., Belyaev A.K. Alekseeva E.L., Polyanskiy A.M., Tretyakov D.A., Yakovlev Yu.A. Phenomenon of skin effect in metals due to hydrogen absorption. Continuum Mech. Thermodyn., 2019, vol. 31, iss. 6, pp. 1-15. DOI: 10.1007/s00161-019-00839-2.

38. Martinsson A., Sandstrom R. Hydrogen depth profile in phosphorus-doped, oxygen-free copper after cathodic charging. Journal of Materials Science, 2012, vol. 47, no. 19, pp. 6768-6776. DOI: $10.1007 / \mathrm{s} 10853-012-6592-y$. 
Подана в журнал: 29.08.2019

УДК 539.3

DOI: $10.17804 / 2410-9908.2019 .5 .006-022$

\title{
СРАВНЕНИЕ УСЛОВИЙ ПЛАСТИЧНОСТИ ДЛЯ МИКРОПОЛЯРНЫХ СРЕД НА ПРИМЕРЕ МОДЕЛИ ЦИЛИНДРИЧЕСКОГО ОБРАЗЦА
}

\author{
К. П. Фролова ${ }^{\text {a)* }}$, Е. Н. Вильчевская ${ }^{6)}$ \\ Институт проблем машиноведения РАН, \\ о. Васильевский, Большой проспект, 61, Санкт-Петербург, Российская Федерация \\ a) iD https://orcid.org/0000-0003-0376-4463 kspfrolova@gmail.com; \\ б) (iD https://orcid.org/0000-0002-5173-3218 vilchevska@ gmail.com \\ *Ответственный автор. Электронная почта: kspfrolova@gmail.com
} Адрес для переписки: о. Васильевский, Большой проспект, 61, 199178, Санкт-Петербург, Российская Федерация Тел.: +7-981-803-37-56; факс: +7-812-321-47-71

В статье рассматриваются условия пластичности, обобщенные различными способами на случай микрополярных сред, с целью исследования эффекта локализации пластических деформаций в металлах. В качестве примера в статье рассматрено полученное в рамках микрополярной теории сплошных сред неоднородное напряженно-деформированное состояние цилиндрического образца, на боковой поверхности которого задан распределенный момент и к торцам которого приложено растягивающее усилие. Оказывается, что пластическое течение инициируется непосредственно у границы образца, в то время как материал в целом продолжает вести себя упруго. Результаты свидетельствуют о необходимости учета моментных взаимодействий между частицами среды при рассмотрении приграничной области, размеры которой сопоставимы с характерным размером структурной неоднородности материала.

Ключевые слова: критерий текучести для микрополярной среды, размерный эффект, псевдоконтинуум Коссера, моментное взаимодействие.

\section{1. Введение}

Согласно имеющимся в литературе данным, реакция материала на нагружение определяется прежде всего процессами, происходящими на мезоуровне, являющимся промежуточным между микро- и макромасштабным $[1,2]$. При этом может наблюдаться локализация пластических деформаций, возникающих в малых по сравнению с объемом тела областях. Так, согласно [3], некоррелированное движение индивидуальных дислокаций в высокопрочных металлах приводит к возникновению локальных зон пластического течения, размеры которых сопоставимы с размерами ядер дислокаций. В работах [4-6] отмечается, что именно в поверхностном слое материала наблюдается широкий спектр атомных конфигураций и большое количество вакансий, приводящих к ослаблению межатомных взаимодействий и развитию интенсивной пластической деформации. В [4, 7] подчеркивается, что поверхностный слой в материале является самостоятельной подсистемой, играющей принципиально важную роль в развитии пластических деформаций.

Для учета структурной неоднородности материала, проявляющейся на мезоуровне, представляется обоснованным применение микрополярной теории, учитывающей, в общем случае, помимо трансляционных вращательные степени свободы элементов среды и вводящей моментные взаимодействия между ними наряду с силовыми [8-11]. Действительно, согласно [12], в материалах с большими градиентами напряжений и наличием высокодефектных субструктур в ходе деформации возможно развитие поворотов отдельных областей, вызванных миграцией границ зерен. Применение микрополярной теории приводит 
к возрастанию вклада моментных напряжений и существенному отличию результата от полученного в рамках классической теории для области концентрации напряжений, сопоставимой с характерным размером структурной неоднородности материала $[13,14]$. При этом вне данной области моментные взаимодействия можно считать малыми второго порядка по сравнению с силовыми. Как следствие, микрополярная теория позволяет описать начало пластического течения, локализованного в области концентрации напряжений, определяемой характерными длинами для деформации изгиба и кручения - параметрами материала, отражающими так называемый «размерный эффект» и сопоставимыми с размерами элементов структуры материала. Сам эффект заключается в том, что в рамках обобщенных сред жесткости на изгиб и кручение у стержней и пластинок при уменьшении их размеров возрастают, в отличие от постоянных значений, предсказываемых классической теорией сплошных сред $[13,14]$.

Основы микрополярной теории пластичности были заложены в [15-20]. Обзор развития теории можно найти в [14, 21-22]. Определение условия пластичности классической теории течения может быть обобщено на случай микрополярных сред двумя способами $[13,14,21]$. Используется либо один общий критерий пластичности, в который входят и силовые, и моментные напряжения $[13,18,20,23,24]$, либо два отдельных критерия для силовых и моментных напряжений соответственно $[13,21]$. Само условие пластичности в обоих случаях заключается в том, что функция текучести становится неотрицательной. Данная функция зависит только от обобщенной интенсивности напряжений, которая в свою очередь может определяться различными способами, как в случае единого критерия, так и в случае двух различных критериев. Наиболее известные формулировки представлены в следующем разделе. Различие состоит в том, каким образом задается набор материальных параметров, фигурирующих в выражении для интенсивности. Часть из них может обнуляться, а часть - приравниваться друг другу.

Интерес представляет сравнение интенсивностей напряжений, определяемых в рамках классической и микрополярной теорий сплошных сред, для понимания степени влияния моментного взаимодействия между частицами среды на поведение материала. В работе в качестве примера будет представлено сравнение интенсивностей напряжений, возникающих при растяжении металлического образца, описываемого классической моделью и моделью псевдоконтинуума Коссера, являющейся частным случаем микрополярной среды. Модель псевдоконтинуума Коссера основана на предположении о том, что взаимодействие между частицами среды осуществляется как за счет силовых, так и за счет моментных напряжений, но при этом дополнительная вращательная степень свободы частиц среды отсутствует, а их повороты определяются через ротор векторного поля перемещений [11]. Помимо этого для модели псевдоконтинуума Коссера в работе будет предложено сравнение интенсивностей напряжений, полученных при использовании различных критериев пластичности, представленных в литературе.

\section{2. Постановка задачи и методы решения}

\section{1. Критерии пластичности для микрополярных сред}

Из классической теории сплошных сред известно, что, во-первых, для изотропного материала условие пластичности не должно зависеть от выбора направления и может быть выражено в виде функции главных инвариантов тензора напряжений. Во-вторых, как правило, всестороннее сжатие не приводит к пластическим деформациям, а потому данная функция зависит от инвариантов девиаторной части тензора напряжений. Наиболее приемлемыми и широко используемыми критериями в классической теории являются критерии Треска и Мизеса $[25,26]$. 
Критерий Мизеса может быть обобщен на микрополярные среды с помощью одного или двух условий пластичности. В общем виде единый критерий, учитывающий влияние как силовых, так и моментных напряжений, вводится следующим образом $[13,14]$ :

$$
\begin{gathered}
Y(\mathbf{T}, \mathbf{M}) \equiv J_{2}(\mathbf{T}, \mathbf{M})-Y_{p}=0, \\
J_{2}(\mathbf{T}, \mathbf{M})=\sqrt{a_{1} \mathbf{S}: \mathbf{S}+a_{2} \mathbf{S} \cdot \mathbf{S}+b_{1} \mathbf{M}: \mathbf{M}+b_{2} \mathbf{M} \cdot \cdot \mathbf{M}},
\end{gathered}
$$

где $\mathbf{S}=\mathbf{T}-1 / 3 \operatorname{tr} \mathbf{T E}$ - девиатор тензора силовых напряжений $\mathbf{T} ; \mathbf{E}-$ единичный тензор, М - тензор моментных напряжений; $a_{1}, a_{2}, \mathrm{~b}_{1}, \mathrm{~b}_{2}$ - материальные параметры; $Y_{p}$ - предел текучести. Двойные скалярные произведения вводятся по следующим правилам: $\mathbf{a b}: \mathbf{c d}=(\mathbf{a} \cdot \mathbf{c})(\mathbf{b} \cdot \mathbf{d}) ; \mathbf{a b} \cdot \mathbf{c d}=(\mathbf{b} \cdot \mathbf{c})(\mathbf{a} \cdot \mathbf{d})$.

Другой возможный способ обобщения критерия Мизеса заключается в постановке двух условий пластичности, представляющих собой две функции, каждая из которых зависит только от одного тензора напряжений (силового или моментного). Соответствующие формулы для функций текучести и интенсивностей напряжений примут вид $[13,14]$ :

$$
\begin{gathered}
Y_{\sigma}(\mathbf{T}) \equiv J_{2}(\mathbf{T})-Y_{p}^{\sigma}=0, Y_{M}(\mathbf{M}) \equiv J_{2}(\mathbf{M})-Y_{p}^{M}=0 \\
J_{2}(\mathbf{T})=\sqrt{a_{1} \mathbf{S}: \mathbf{S}+a_{2} \mathbf{S} \cdot \mathbf{S}}, J_{2}(\mathbf{M})=\sqrt{b_{1} \mathbf{M}: \mathbf{M}+b_{2} \mathbf{M} \cdot \cdot \mathbf{M}} .
\end{gathered}
$$

В этом случае для силовых и моментных напряжений вводятся два различных предела текучести: $Y_{p}^{\sigma}$ и $Y_{p}^{M}$ соответственно.

Введенные формулами (1) и (3) критерии должны совпадать с классическим при переходе к безмоментной среде $\left(\mathbf{M}=0 ; \mathbf{T}=\mathbf{T}^{T}\right.$, где верхний индекс $T$ означает транспонирование), что накладывает ограничение на материальные параметры $a_{1}$, ѐ $a_{2}$, а именно: $a_{1}+a_{2}=1 / 2$. Это единственное ограничение не дает однозначного определения дополнительных материальных констант, входящих в выражения для интенсивностей, описываемых формулами (2) и (4). Стоит отметить, что определение данных констант является одной из основных задач при применении неклассических сплошных сред $[13,14,19]$. Не останавливаясь на способах получения того или иного набора значений данных параметров, приведем наиболее широко известные из них.

Авторы [18-20, 27-28] вводят следующий единый критерий текучести, в котором $\mathrm{b}_{2}=0$ :

$$
J_{2}(\mathbf{T}, \mathbf{M})=\sqrt{a_{1} \mathbf{S}: \mathbf{S}+a_{2} \mathbf{S} \cdot \mathbf{S}+b_{1} \mathbf{M}: \mathbf{M}}
$$

Остальные параметры определяются различными способами. Под «стандартным» набором предполагаются значения $a_{1}=a_{2}=1 / 4, \mathrm{~b}_{1}=1 /\left(2 l^{2}\right)$, где $l$ - характерный параметр материала размерности длины, существенно влияющий на размер области локализации концентрации напряжений $[13,20,24]$. В $[18,27]$ при решении статической задачи используются значения $a_{1}=3 / 4, a_{2}=-1 / 4, \mathrm{~b}_{1}=1 /\left(8 l^{2}\right)$. В [20] показано, что выбор данных значений не оказывает существенного влияния на переход в пластическое состояние.

Авторы $[17,21]$ вводят интенсивность для случая единого критерия текучести как 


$$
J_{2}(\mathbf{T}, \mathbf{M})=\sqrt{\frac{1}{2} \mathbf{S}: \mathbf{S}+\frac{1}{2}\left(\beta^{2}-1\right) \boldsymbol{\sigma}^{A}: \boldsymbol{\sigma}^{A}+b_{1} \mathbf{M}: \mathbf{M}}
$$

Для случая двух различных критериев для силовых и моментных напряжений автор [21] предлагает следующие выражения для определения соответствующих им интенсивностей:

$$
J_{2}(\boldsymbol{\sigma})=\sqrt{\frac{1}{2} \mathbf{S}: \mathbf{S}+\frac{1}{2}\left(\beta^{2}-1\right) \boldsymbol{\sigma}^{A}: \boldsymbol{\sigma}^{A}}, J_{2}(\mathbf{M})=\sqrt{b_{1} \mathbf{M}: \mathbf{M}} .
$$

В выражениях (6) и (7) принимается, что $\mathrm{b}_{1}=(\alpha / L)^{2}, \alpha, \beta \geq 0$ - безразмерные материальные параметры материала; $L$ - масштабный параметр размерности длины.

При $\beta=0$ интенсивность, определяемая формулой (6), совпадает с введенной в [22, 29, 30]. Тогда выражение (6) совпадает с выражением (2), а выражение (7) - с выражением (4) при следующих значениях материальных параметров: $a_{2}=\mathrm{b}_{2}=0 ; \mathrm{a}_{1}=1 / 2 ; \mathrm{b}_{1}=(\alpha / L)^{2}$.

Заметим, что при растяжении образца интенсивность напряжений для всех выше перечисленных случаев определяется как

$$
\tilde{J}_{2}=\sqrt{3} J_{2}
$$

При определении интенсивности напряжений будем использовать в выражениях (2) и (4) наборы параметров, представленные в таблице. При этом в качестве масштабного параметра $l$ размерности длины будем использовать характерную длину для деформации изгиба, как это предложено например в [28].

Таблица - Наборы параметров в выражениях для интенсивности напряжений

\begin{tabular}{|c|c|}
\hline № & Значения параметров \\
\hline 1 & $a_{1}=a_{2}=\frac{1}{4} ; \mathrm{b}_{1}=\frac{1}{2 l_{b}^{2}} ; \mathrm{b}_{2}=0$ \\
\hline 2 & $a_{1}=\frac{3}{4} ; a_{2}=-\frac{1}{4} ; \mathrm{b}_{1}=\frac{1}{8 l_{b}^{2}} ; \mathrm{b}_{2}=0$ \\
\hline 3 & $a_{1}=\frac{1}{2} ; a_{2}=0 ; \mathrm{b}_{1}=\frac{1}{2 l_{b}^{2}} ; \mathrm{b}_{2}=0$ \\
\hline 4 & $a_{1}=a_{2}=\frac{1}{4} ; \mathrm{b}_{1}=\mathrm{b}_{2}=\frac{1}{2 l_{b}^{2}}$ \\
\hline 5 & $a_{1}=a_{2}=\frac{1}{4} ; \mathrm{b}_{1}=\mathrm{b}_{2}=\frac{1}{4 l_{b}^{2}}$ \\
\hline
\end{tabular}

\section{2. Задача о растяжении цилиндрического образца}

В качестве примера определим по приведенным в предыдущем подразделе формулам интенсивность напряжений при растяжении металлического образца цилиндрической формы радиуса $r_{0}$, на боковой поверхности которого задан распределенный момент $-M_{0} \mathbf{e}_{\varphi}$. Напряженно-деформированное состояние образца получено в [31] в рамках теории упругости 
псевдоконтинуума Коссера при решении задачи в цилиндрической системе координат $(r, \varphi, \mathrm{z})$ с помощью асимптотического метода. Полученные силовые и моментные напряжения имеют вид:

$$
\begin{gathered}
\mathbf{T}=\frac{F}{S} \mathbf{e}_{z} \mathbf{e}_{z}+2 \sqrt{\frac{\mu}{\beta_{3}}} M_{0} e^{-2 \sqrt{\frac{\mu}{\beta_{3}}}\left(r_{0}-r\right)} \mathbf{e}_{z} \mathbf{e}_{r} ; \\
\mathbf{M}=-M_{0} e^{-2 \sqrt{\frac{\mu}{\beta_{3}}}\left(r_{0}-r\right)}\left(\frac{\beta_{2}}{\beta_{3}} \mathbf{e}_{\varphi} \mathbf{e}_{r}+\mathbf{e}_{r} \mathbf{e}_{\varphi}\right),
\end{gathered}
$$

где $F$ - растягивающая сила, приложенная к торцам цилиндра; $S=\pi r_{0}^{2}-$ площадь поперечного сечения цилиндра; $\mu$ - модуль сдвига; $\beta_{2}, \beta_{3}$ - неклассические упругие модули, отражающие вклад моментных взаимодействий между частицами среды.

Определение значений неклассических модулей, фигурирующих в выражениях (9) и (10), сопряжено с техническими сложностями, поскольку измерить данные параметры напрямую подобно классическим упругим модулям невозможно. Для оценки данных модулей используются методы размерных эффектов, при которых значения модулей определяются на основании сопоставления результатов измерений с полученными расчетными зависимостями жесткостей на изгиб и кручение от размеров образца [14]. Несмотря на значительный прогресс в области микрополярной теории сплошных сред, неклассические упругие модули на данный момент определены лишь для некоторых материалов [32-34].

Таким образом, в модель микрополярной среды можно ввести характерные длины для деформации изгиба $l_{b}$ и кручения $l_{t}$, которые связаны с упругими модулями следующим образом [9]:

$$
l_{b}=\sqrt{\frac{\beta_{3}}{4 \mu}} ; l_{t}=\sqrt{\frac{\beta_{2}+\beta_{3}}{2 \mu}} .
$$

При этом значения неклассических упругих модулей ограничены условием: $-\beta_{3} \leq \beta_{2} \leq \beta_{3}$, что накладывает аналогичное ограничение и на значения характерных длин, а именно: $0 \leq l_{t} \leq 2 l_{b}$.

Из приведенного в формулах (9) и (10) решения видно, что напряженно-деформированное состояние образца у его боковой поверхности может быть уточнено с помощью модели, предложенной в рамках микрополярной теории упругости, тогда как при удалении от поверхности эффект, связанный с наличием распределенного момента на границе, исчезает. Таким образом, в приграничной области возникает концентрация напряжений, что может инициировать локальное пластическое течение.

\section{3. Результаты и обсуждение}

Выпишем все двойные скалярные произведения, фигурирующие в выражениях для интенсивности напряжений, представленных формулами (2) и (4):

$$
\mathbf{S}: \mathbf{S}=\frac{2}{3} \frac{F^{2}}{S^{2}}+\frac{M_{0}^{2}}{l_{b}^{2}} e^{-2 \frac{\left(r_{0}-r\right)}{l_{b}}}
$$




$$
\begin{gathered}
\mathbf{S} \cdot \mathbf{S}=\frac{2}{3} \frac{F^{2}}{S^{2}} \\
\mathbf{M}: \mathbf{M}=\frac{\left(l_{t}^{2}-2 l_{b}^{2}\right)^{2}+4 l_{b}^{4}}{4 l_{b}^{4}} M_{0}^{2} e^{-2 \frac{\left(r_{0}-r\right)}{l_{b}}} ; \\
\mathbf{M} \cdot \mathbf{M}=\frac{l_{t}^{2}-2 l_{b}^{2}}{l_{b}^{2}} M_{0}^{2} e^{-2 \frac{\left(r_{0}-r\right)}{l_{b}}} .
\end{gathered}
$$

При решении задачи в рамках классической теории сплошных сред моментные напряжения равны нулю, а в выражении для силовых напряжений отсутствует компонента, содержащая экспоненциальную функцию. Тогда классическая интенсивность напряжений при растяжении будет определяться формулой (16):

$$
\tilde{J}_{2}^{c l}=\sqrt{\frac{F^{2}}{S^{2}}}
$$

Для получения численных результатов рассмотрим цилиндрический образец радиуса $r_{0}=0,004$ і̀ и длины $L=0,04$ і̀ , выполненный из атмосферостойкой стали с модулем сдвига $\mu=80000$ İ Ï à. Примем для определенности, что распределенный момент на границе $M_{0}=64000$ İ à. Предел текучести при растяжении находится в диапазоне $Y_{p}=465-640$ МПа. Примем, что растягивающее усилие $F=20000$ Í . Тогда, согласно выражению (16), интенсивность напряжений окажется равной $\tilde{J}_{2}^{c l} \approx 400 \mathrm{MПа,} \mathrm{что} \mathrm{не} \mathrm{превышает} \mathrm{значения} \mathrm{предела}$ текучести при растяжении. Таким образом, при моделировании поведения материала в рамках безмоментной теории оно является чисто упругим.

В работе [31] предложен метод определения материальной константы $l_{b}$, основанный на сравнении экспериментальных и аналитических данных. При этом предполагается, что модель объясняет существенно неоднородное распределение водорода, проникающего в металл из внешней среды. Так, экспериментальные данные свидетельствуют о том, что водород практически весь аккумулируется в тонком поверхностном слое [35-38]. Экспериментально измеренная толщина пограничного слоя $r_{*}$, содержащего избыток водорода, сравнивается с аналитически полученной шириной области с дополнительными продольными смещениями частиц континуума. Тогда имеет место следующее соотношение: $l_{b}=r_{0}\left(1-r_{*} / r_{0}\right) / \ln k$, где $k$ показывает степень затухания продольных перемещений при удалении от боковой поверхности образца на толщину пограничного слоя. При $k=100$ получаем $l_{b}=13 \mu$.

\section{1. Единый критерий пластичности}

При использовании стандартного набора параметров (таблицы № 1) в выражении для интенсивности напряжений, обобщенной на случай микрополярного материала формулой (2), получаем следующее равенство:

$$
\tilde{J}_{2_{1}}=\sqrt{\frac{F^{2}}{S^{2}}+\frac{3}{8} \frac{M_{0}^{2}}{l_{b}^{6}}\left(\left(l_{t}^{2}-2 l_{b}^{2}\right)^{2}+6 l_{b}^{4}\right) e^{-2 \frac{\left(r_{0}-r\right)}{l_{b}}}} .
$$


При использовании набора параметров, предложенного в $[18,27]$ для статической модели (таблица № 2), интенсивность напряжений, вычисленная по формуле (2), оказывается равной

$$
\tilde{J}_{2_{2}}=\sqrt{\frac{F^{2}}{S^{2}}+\frac{3}{32} \frac{M_{0}^{2}}{l_{b}^{6}}\left(\left(l_{t}^{2}-2 l_{b}^{2}\right)^{2}+28 l_{b}^{4}\right) e^{-2 \frac{\left(r_{0}-r\right)}{l_{b}}}} .
$$

При использовании набора параметров № 3 (таблица) общая интенсивность определяется выражением

$$
\tilde{J}_{2_{3}}=\sqrt{\frac{F^{2}}{S^{2}}+\frac{3 M_{0}^{2}}{8 l_{b}^{6}}\left(\left(l_{t}^{2}-2 l_{b}^{2}\right)^{2}+8 l_{b}^{4}\right) e^{-2 \frac{\left(r_{0}-r\right)}{l_{b}}}} .
$$

Найдем функции интенсивности, определяемые формулами (17)-(19), для следующих трех значений характерной длины для деформации кручения: $l_{t}=0\left(\beta_{2}=-\beta_{3}\right) ; l_{t}=\sqrt{2} l_{b}\left(\beta_{2}=0\right)$; $l_{t}=2 l_{b}\left(\beta_{2}=\beta_{3}\right)$. При сравнении выражений (17)-(19) видно, что имеют место следующие равенства: $\left.\quad J_{2_{1}}\right|_{l_{t}=0}=\left.J_{2_{1}}\right|_{l_{t}=2 l_{b}} ;\left.\quad J_{2_{2}}\right|_{l_{t}=0}=\left.J_{2_{2}}\right|_{l_{t}=2 l_{b}} ;\left.J_{2_{3}}\right|_{l_{t}=0}=\left.J_{2_{3}}\right|_{l_{t}=2 l_{b}}$. Интенсивность напряжений на боковой поверхности образца принимает значения $\tilde{J}_{2_{1}}\left(r_{0}\right)=9,5$ Äİ à; $\tilde{J}_{2_{2}}\left(r_{0}\right)=8,5$ Äİ à; $\tilde{J}_{2_{3}}\left(r_{0}\right)=10,5$ Äİ à $\quad-$ при $l_{t}=0, l_{t}=2 l_{b} \quad$ и $\quad$ значения $\tilde{J}_{2_{1}}\left(r_{0}\right)=7,4$ ÄI à; $\tilde{J}_{2_{2}}\left(r_{0}\right)=7,9$ Äİ à; $\tilde{J}_{2_{3}}\left(r_{0}\right)=8,5$ Äİ à - при $l_{t}=\sqrt{2} l_{b}$. Графики зависимости интенсивностей от безразмерной радиальной координаты $\left(x=1-r / r_{0}\right)$ для разных значений характерной длины для деформации кручения представлены на рис. 1.

При анализе результата, представленного на рис. 1, видно, что учет моментных взаимодействий между частицами микрополярной среды приводит к возникновению пластического течения непосредственно у боковой поверхности образца, поскольку интенсивность напряжений в этой области значительно превышает предел текучести при растяжении. При удалении от границы интенсивность стремительно убывает и стремится к полученной в рамках классической теории. Следовательно, уточненное с помощью микрополярной теории решение играет роль только в тонком поверхностном слое, толщина которого сопоставима с размерами структурной неоднородности материала. При выходе же за пределы данной области моментные взаимодействия можно считать малыми второго порядка по сравнению с силовыми.

Как видно из представленных на рис. 1 графиков, значение характерной длины для деформации кручения влияет на значение интенсивности у боковой поверхности образца. Характер же убывания интенсивности до значения, получаемого в рамках классической теории, равно как и ширина зоны локализации концентрации напряжений, не меняются существенно при изменении данного параметра.

Заметим, что качественно результаты, полученные для разных наборов параметров в выражении для интенсивности, не отличаются друг друга. Тем не менее имеются количественные отличия, во-первых, в максимальном значении интенсивности, а во-вторых, в ширине зоны локализации пластического течения (рис. 2). 


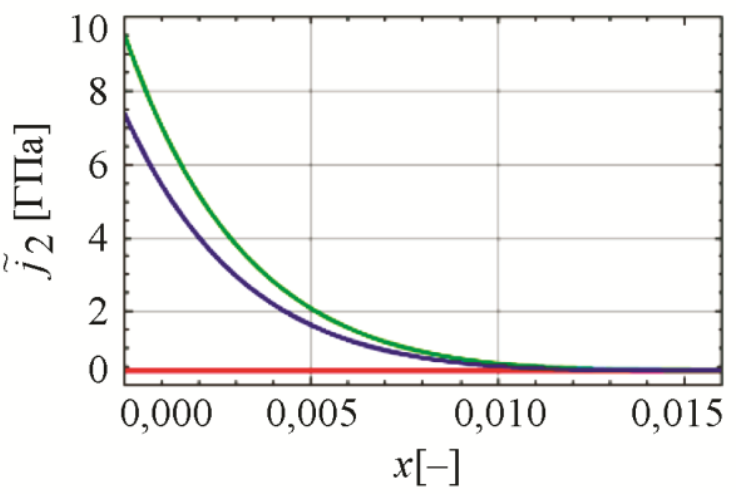

$a$

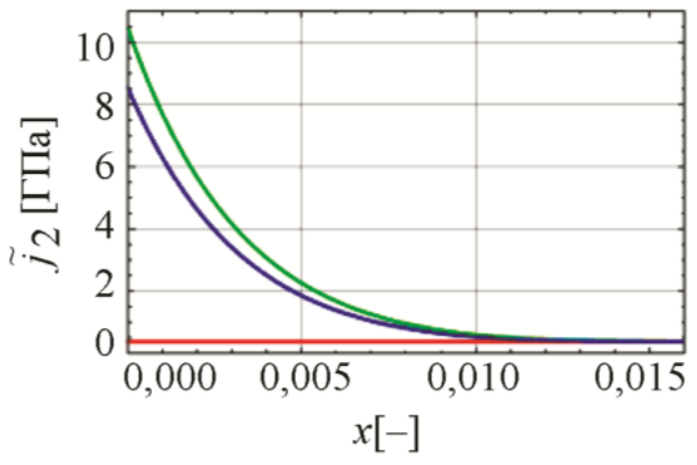

B

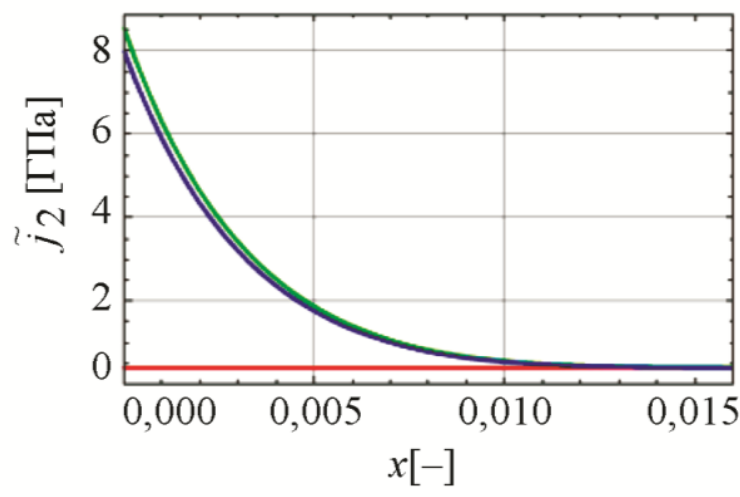

$\sigma$

- Классическая среда;

- Микрополярная среда, $l_{t}=0$ и $l_{t}=2 l_{b}$;

-Микрополярная среда, $l_{t}=\sqrt{ } 2 l_{b}$

Рис. 1. Зависимости интенсивности напряжений от безразмерной радиальной координаты при разных значениях $l_{t}: a-\tilde{J}_{2_{1}}(x) ; \sigma-\tilde{J}_{2_{2}}(x) ; в-\tilde{J}_{2_{3}}(x)$

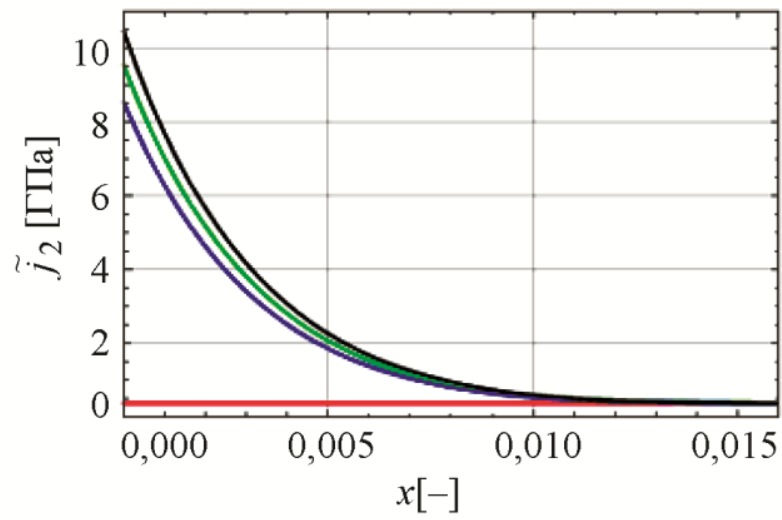

— Классическая среда;

— Микрополярная среда, $\tilde{j}_{22}$;

$a$

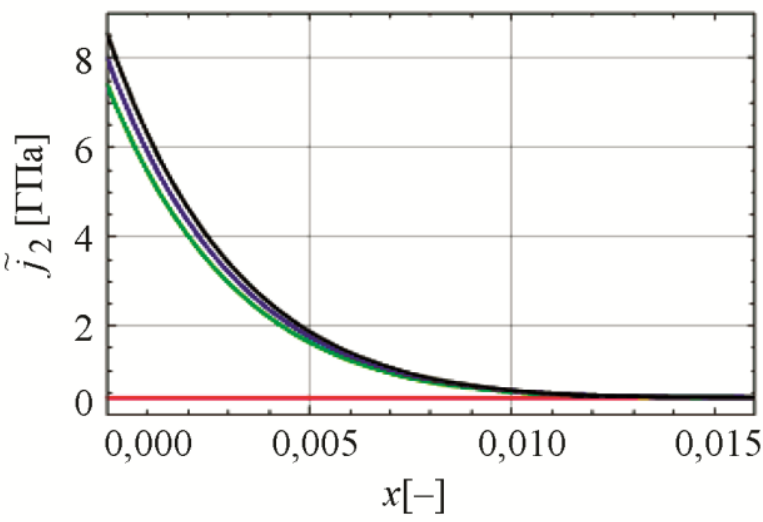

- Микрополярная среда, $\tilde{j}_{2}$;

— Микрополярная среда, $\tilde{j}_{23}$;

$\sigma$

Рис. 2. Сравнение интенсивностей напряжений, полученных для разных наборов параметров (таблица № 1-3): $a-l_{t}=0, l_{t}=2 l_{b} ; \sigma-l_{t}=\sqrt{2} l_{b}$

Рассмотрим теперь общее выражение для интенсивности, в котором фигурируют все двойные скалярные произведения, представленные формулами (12)-(15). Остановимся 
на двух последних наборах параметров, представленных в таблице. В первом случае (таблица № 4) имеем следующее выражение для обобщенной интенсивности:

$$
\tilde{J}_{2_{4}}=\sqrt{\frac{F^{2}}{S^{2}}+\frac{3 M_{0}^{2}}{16 l_{b}^{6}}\left(l_{t}^{4}+4 l_{b}^{4}\right) e^{-2 \frac{\left(r_{0}-r\right)}{l_{b}}}} ;
$$

во втором случае:

$$
\tilde{J}_{2_{5}}=\sqrt{\frac{F^{2}}{S^{2}}+\frac{3 M_{0}^{2}}{8 l_{b}^{6}}\left(l_{t}^{4}+2 l_{b}^{4}\right) e^{-2 \frac{\left(r_{0}-r\right)}{l_{b}}}} .
$$

При сравнении выражений (20) и (21) видно, что при $l_{t}=0$ значения интенсивностей совпадают. Сравнение значений интенсивностей, вычисленных по формулам (20) и (21), для $l_{t}=0 ; l_{t}=\sqrt{2} l_{b}$ и $l_{t}=2 l_{b}$ представлено на рис. 3 .

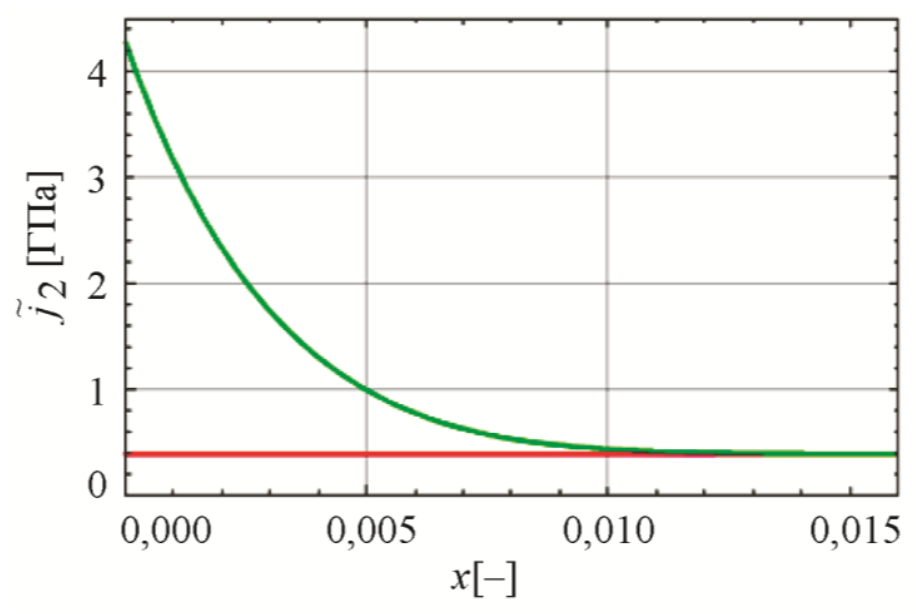

$a$
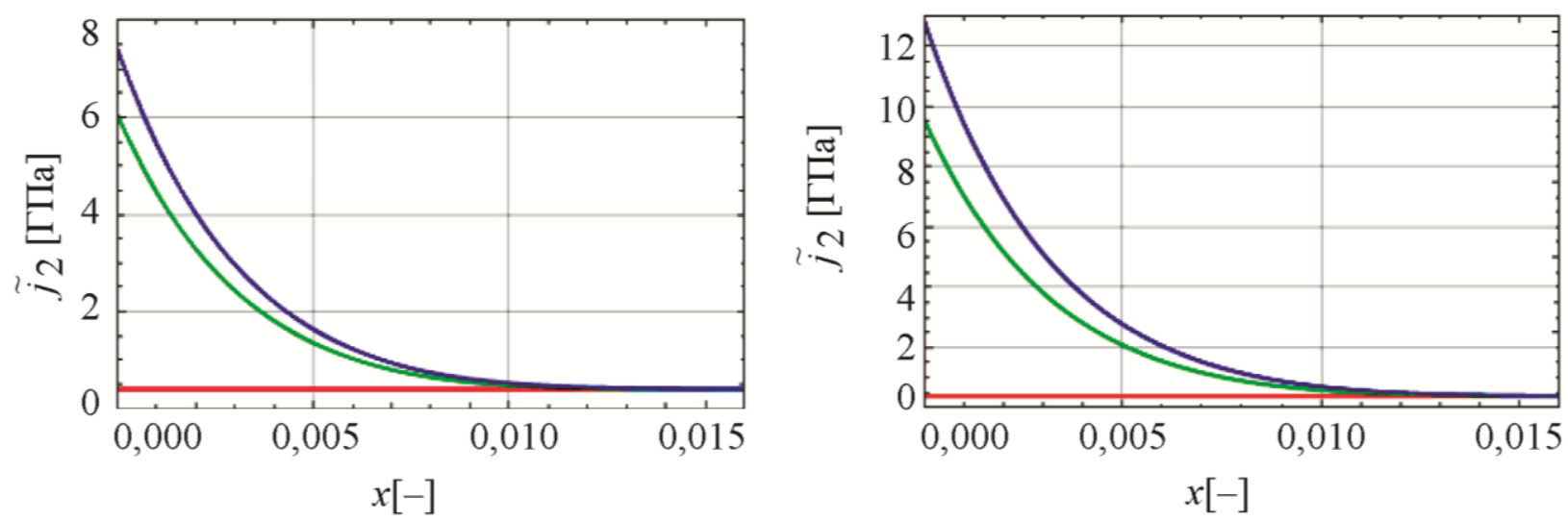

- Классическая среда; - Микрополярная среда, $\widetilde{j}_{2} ; \quad-$ Микрополярная среда, $\tilde{j}_{2}$ $\sigma$

Рис. 3. Сравнение интенсивностей напряжений, учитывающих все двойные скалярные произведения между тензорами силовых и моментных напряжений:

$$
a-l_{t}=0 ; \sigma-l_{t}=\sqrt{2} l_{b} ; в-l_{t}=2 l_{b}
$$


При анализе результатов, представленных на рис. 3, и сравнении их с полученными выше можно заметить, что качественного изменения картины не наблюдается при использовании большего или меньше числа ненулевых параметров, фигурирующих в выражении для обобщенной интенсивности. Основное различие заключается в величине, определяющей интенсивность напряжений в области их концентрации. При этом очевидно, что все предложенные значения существенно превышают предел текучести материала при растяжении. Таким образом, принципиально важно учитывать моментное взаимодействие при определении поведения материала в области, соразмерной с масштабом структурной неоднородности.

\section{2. Два критерия пластичности. Сравнение с единым критерием}

Использование наборов параметров, перечисленных в таблице, даст следующие выражения для интенсивностей силовых и моментных напряжений соответственно:

$$
\begin{aligned}
& \tilde{J}_{2_{1}}(\mathbf{T})=\sqrt{\frac{F^{2}}{S^{2}}+\frac{3}{4} \frac{M_{0}^{2}}{l_{b}^{2}} e^{-2 \frac{\left(r_{0}-r\right)}{l_{b}}}} ; \tilde{J}_{2_{1}}(\mathbf{M})=\sqrt{\frac{3 M_{0}^{2}}{8 l_{b}^{6}}\left(\left(l_{t}^{2}-2 l_{b}^{2}\right)^{2}+4 l_{b}^{4}\right) e^{-2 \frac{\left(r_{0}-r\right)}{l_{b}}}} ; \\
& \tilde{J}_{2_{2}}(\mathbf{T})=\sqrt{\frac{F^{2}}{S^{2}}+\frac{9}{4} \frac{M_{0}^{2}}{l_{b}^{2}} e^{-2 \frac{\left(r_{0}-r\right)}{l_{b}}}} ; \tilde{J}_{2_{2}}(\mathbf{M})=\sqrt{\frac{3 M_{0}^{2}}{32 l_{b}^{6}}\left(\left(l_{t}^{2}-2 l_{b}^{2}\right)^{2}+4 l_{b}^{4}\right) e^{-2 \frac{\left(r_{0}-r\right)}{l_{b}}}} ; \\
& \tilde{J}_{2_{3}}(\mathbf{T})=\sqrt{\frac{F^{2}}{S^{2}}+\frac{3}{2} \frac{M_{0}^{2}}{l_{b}^{2}} e^{-2 \frac{\left(r_{0}-r\right)}{l_{b}}}} ; \tilde{J}_{2_{3}}(\mathbf{M})=\sqrt{\frac{3 M_{0}^{2}}{8 l_{b}^{6}}\left(\left(l_{t}^{2}-2 l_{b}^{2}\right)^{2}+4 l_{b}^{4}\right) e^{-2 \frac{\left(r_{0}-r\right)}{l_{b}}}} ; \\
& \tilde{J}_{2_{4}}(\mathbf{T})=\sqrt{\frac{F^{2}}{S^{2}}+\frac{3}{4} \frac{M_{0}^{2}}{l_{b}^{2}} e^{-2 \frac{\left(r_{0}-r\right)}{l_{b}}}} ; \tilde{J}_{2_{4}}(\mathbf{M})=\frac{\sqrt{3} M_{0} l_{t}^{2}}{2 \sqrt{2} l_{b}^{3}} e^{-\frac{\left(r_{0}-r\right)}{l_{b}}} ; \\
& \tilde{J}_{2_{5}}(\mathbf{T})=\sqrt{\frac{F^{2}}{S^{2}}+\frac{3}{4} \frac{M_{0}^{2}}{l_{b}^{2}} e^{-2 \frac{\left(r_{0}-r\right)}{l_{b}}}} ; \tilde{J}_{2_{5}}(\mathbf{M})=\frac{\sqrt{3} M_{0} l_{t}^{2}}{4 l_{b}^{3}} e^{-\frac{\left(r_{0}-r\right)}{l_{b}}}
\end{aligned}
$$

При сравнении выражений (22)-(26) отметим, что в некоторых случаях для разных наборов параметров совпадают либо интенсивности силовых ((22), (25) и (26)), либо интенсивности моментных напряжений ((22) и (24)). Интерес представляет сравнение данных интенсивностей с соответствующими интенсивностями, полученными при использовании единого условия пластичности.

Поскольку влияние значения параметра характерной длины для деформации кручения на величину интенсивности было обсуждено в предыдущем пункте, при рассмотрении критериев пластичности в данном разделе примем для определенности, что $l_{t}=\sqrt{2} l_{b}$.

Графики зависимостей интенсивностей от безразмерной радиальной координаты для случая одного и двух критериев пластичности представлены на рис. 4. 




$a$

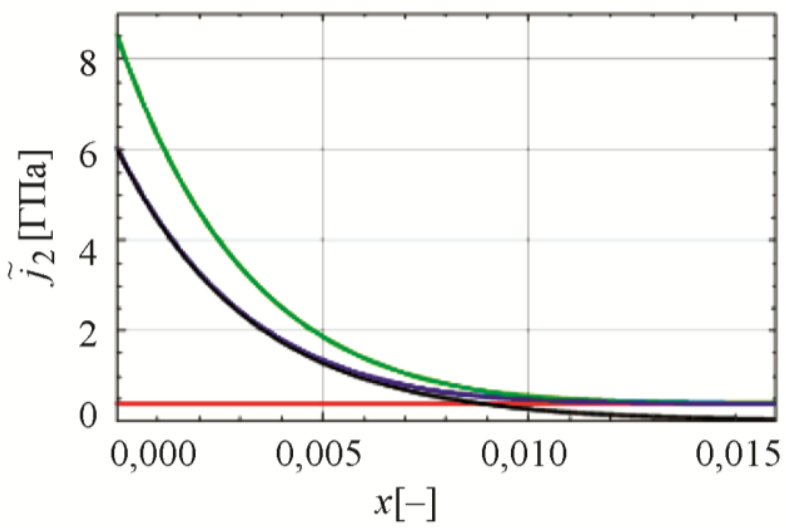

B

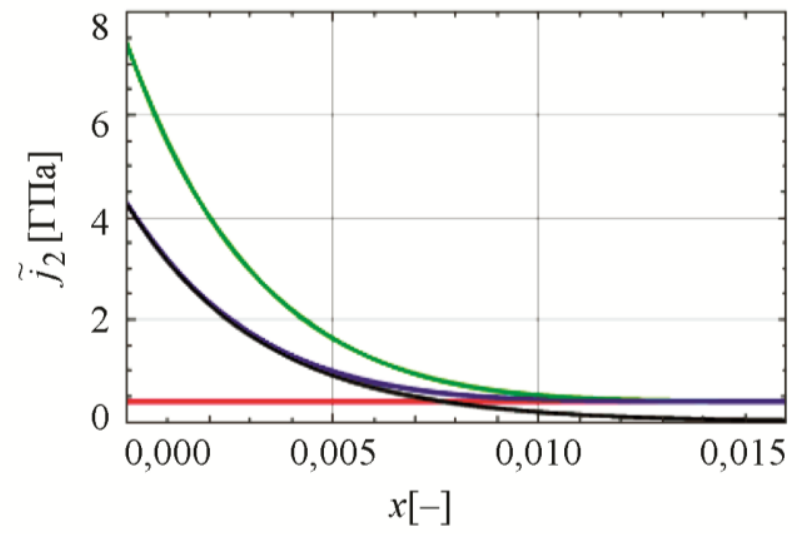

$\partial$

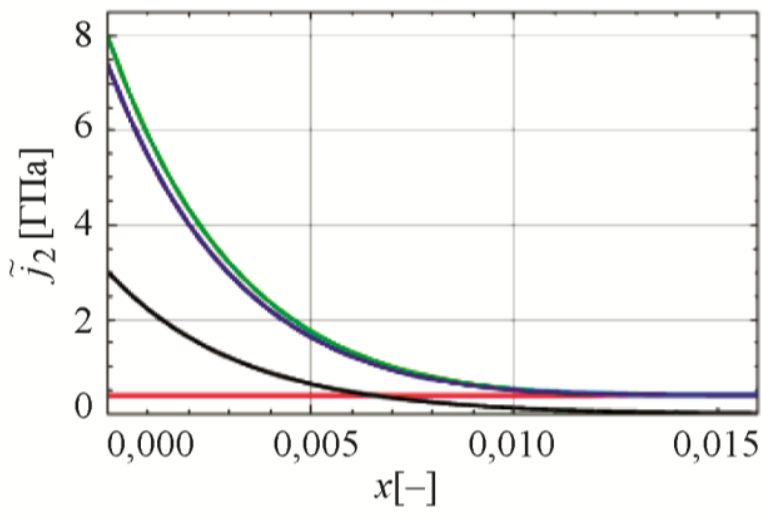

$\sigma$

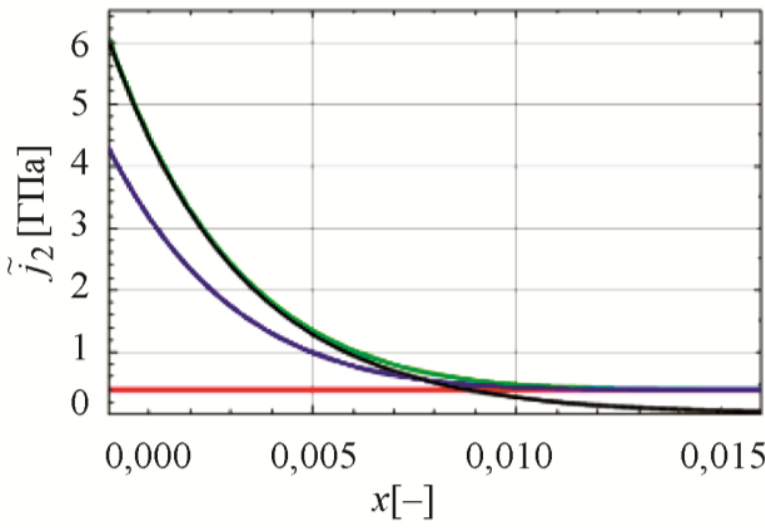

2

- Классическая среда;

- Микрополярная среда, $\tilde{j}_{2}(\mathbf{T}, \mathbf{M})$;

- Микрополярная среда, $\tilde{j}_{2}(\mathbf{T})$;

- Микрополярная среда, $\tilde{j}_{2}(\mathbf{M})$

Рис. 4. Сравнение интенсивностей напряжений, вычисленных для одного и для двух критериев пластичности: $a$ - набор параметров № $1 ; \sigma$ - набор параметров № 2; в - набор параметров № 3; 2 - набор параметров № 4; $\partial$ - набор параметров № 5

Из представленных на рис. 4 графиков видно, что общая интенсивность напряжений, зависящая одновременно и от силовых, и от моментных напряжений, выше значений интенсивностей, зависящих только от одного из типа напряжений. При этом интенсивность моментных напряжений, как и следовало ожидать, быстро убывает при удалении от зоны концентрации напряжений. 


\section{4. Заключение}

В работе представлен анализ неоднородного напряженно-деформированного состояния растягиваемого цилиндрического образца, на боковой поверхности которого задан распределенный момент. В результате учета моментных взаимодействий между частицами среды в рамках микрополярной теории оказывается, что интенсивность напряжений в приграничной зоне значительно превышает предел текучести при растяжении. При этом функция интенсивности резко убывает при удалении от боковой поверхности цилиндрического образца до значения, прогнозируемого классической теорией сплошных сред. Таким образом, при общем упругом поведении материала может наблюдаться локализованное пластическое течение в приграничной области, размеры которой сопоставимы с размерами структурной неоднородности материала.

\section{Литература}

1. Физическая мезомеханика и компьютерное конструирование материалов / отв. ред. В. Е. Панин. - Новосибирск, «Наука», 1995.

2. Панин В. Е., Гриняев Ю. В., Псахье С. Г. Физическая мезомеханика: достижения за два десятилетия развития, проблемы и перспективы // Физическая мезомеханика. - 2004. Vol. 7, no. 4. - C. 93-113.

3. Tyumentsev A. N., Korotayev A. D., Pinzhin Yu. P. Highly Defective Structural States, Fields of Local Internal Stresses and Cooperative Mesoscopic Mechanisms of Crystal Deformation and Reorientation in Nanostructured Metal Materials // Phys. Mesomech. - 2004. - Vol. 7, nos. 3-4. - P. 31-46.

4. Панин В. Е., Панин А. В. Эффект поверхностного слоя в деформируемом твердом теле // Физическая мезомеханика. - 2005. - Vol. 8, no. 5. - С. 7-15.

5. Kuznetsov P. V. and Panin V. E. Direct Observation of Flows of Defects and of nm-Range Localization of Deformation on Duralumin Surface with the Aid of Scanning Tunnel and Atom Force Microscopes // Phys. Mesomech. - 2000. - Vol. 3, no. 2. - P. 85-91.

6. Роль локальной кривизны поверхностного слоя поликристаллов титана в зарождении и развитии усталостного разрушения / В. Е. Панин, Т. Ф. Елсукова, Ю. Ф. Попкова, Ю. И. Почивалов // Вопросы атомной науки и техники. - 2015. - Vol. 96, no. 2. - С. 148-153.

7. On the nature of plastic strain localization in solids. / V. E. Panin, V. E. Egorushkin, A. V. Panin, D. D. Moiseenko // Technical Physics. - 2017. - Vol. 52, iss. 8. - P. 1024-1030. DOI: $10.1134 / \mathrm{S} 1063784207080105$.

8. Eremeyev V. A., Lebedev L. P., Altenbach H. Foundations of Micropolar Mechanics. Springer Science \& Business Media, 2012.

9. Eringen A. C. Theory of micropolar elasticity // Microcontinuum field theories. - Springer, New York, NY, 1999. - P. 101-248. - DOI: 10.1007/978-1-4612-0555-5_5.

10. Mindlin R. D. Micro-structure in linear elasticity // Archive for Rational Mechanics and Analysis. - 1964. - Vol. 16, no. 1. - P. 51-78. - DOI: 10.1007/BF00248490.

11. Mindlin R. D., Tiersten H. F. Effects of couple-stresses in linear elasticity // Archive for Rational Mechanics and analysis. - 1962. - Vol. 11, no. 1. - P. 415-448.

12. Структура и сопротивление деформированию ГЦК ультрамелкозернистых металлов и сплавов / Э. В. Козлов, Н. А. Конева, А. Н. Жданов, Н. А. Попова, Ю. Ф. Иванов // Физическая мезомеханика. - 2004. - Vol. 7, no. 4. - Р. 93-113.

13. Forest S., Sievert R. Elastoviscoplastic constitutive frameworks for generalized continua // Acta Mechanica. - 2003. - Vol. 160, nos. 1-2. - P. 71-111. - DOI: 10.1007/s00707-002-0975-0.

14. Смолин И. Ю. Использование микрополярных моделей для описания пластического деформирования на мезоуровне // Вестник Пермского национального исследовательского политехнического университета. Механика. - 2006. - № 14. - С. 189-205. 
15. Lippmann H. Eine Cosserat-Theorie des plastischen Fließens // Acta Mechanica. - 1969. Vol. 8, nos. 3-4. - P. 255-284. - DOI: 10.1007/BF01182264.

16. Sawczuk A. On the yielding of Cosserat continua // Archives of Mechanics. - 1967. Vol. 19, no. 3. - P. 471-480.

17. Besdo D. Ein beitrag zur nichtlinearen theorie des Cosserat-kontinuums // Acta Mechanica. 1974. - Vol. 20, nos. 1-2. - P. 105-131. - DOI: 10.1007/BF01374965.

18. Mühlhaus H. B., Vardoulakis I. The thickness of shear bands in granular materials // Geotechnique. - 1987. - Vol. 37, no. 3. - P. 271-283. - DOI: 10.1680/geot.1987.37.3.271.

19. De Borst R. Simulation of strain localization: a reappraisal of the Cosserat continuum // Engineering computations. - 1991. - Vol. 8, no. 4. - P. 317-332. - DOI: 10.1108/eb023842.

20. De Borst R. A generalisation of J2-flow theory for polar continua // Computer Methods in Applied Mechanics and Engineering. - 1993. - Vol. 103, no. 3. - P. 347-362. DOI: 10.1016/0045-7825(93)90127-J.

21. Lippmann H. Cosserat Plasticity and Plastic Spin // Appl. Mech. Rev. - 1995. - Vol. 48, no. 11. - DOI: 10.1115/1.3005091.

22. Altenbach H., Eremeyev V. A. Strain rate tensors and constitutive equations of inelastic micropolar materials // International Journal of Plasticity. - 2014. - Vol. 63. - P. 3-17. DOI: 10.1016/j.ijplas.2014.05.009.

23. Dietsche A., Steinmann P., Willam K. Micropolar elastoplasticity and its role in localization // International Journal of Plasticity. - 1993. - Vol. 9, no. 7. - P. 813-831. - DOI: 10.1016/07496419(93)90053-S.

24. Steinmann P. A micropolar theory of finite deformation and finite rotation multiplicative elastoplasticity // International Journal of Solids and Structures. - 1994. - Vol. 31, no. 8. - P. 1063-1084. - DOI: 10.1016/0020-7683(94)90164-3.

25. Качанов Л. М. Основы теории пластичности. - М : Наука, 1969. -420 с.

26. Мейз Дж. Теория и задачи механики сплошных сред : учебник. - М. : Мир, 1974. $319 \mathrm{c}$.

27. Vardoulakis I. Shear-banding and liquefaction in granular materials on the basis of a Cosserat continuum theory // Ingenieur-Archiv. - 1989. - Vol. 59, no. 2. - P. 106-113. DOI: $10.1007 / \mathrm{BF} 00538364$.

28. Salehi S. H., Salehi M. Numerical investigation of nanoindentation size effect using micropolar theory // Acta Mechanica. - 2014. - Vol. 225, no. 12. - P. 3365-3376. DOI: $10.1007 / \mathrm{s} 00707-014-1116-2$.

29. Neuber H. Über Probleme der Spannungskonzentration im Cosserat-Kцrper // Acta Mechanica. - 1966. - Vol. 2, no. 1. - P. 48-69. - DOI: 10.1007/BF01176729.

30. Groen A. E., Schellekens J. C. J., De Borst R. Three-dimensional finite element studies of failure in soil bodies using a Cosserat continuum // Computer Methods and Advances in Geomechanics : Proceedings of the 8th International Conference on Computer Methods and Advances in Geomechanics, Morgantown, West Virginia, USA, 22-28 May 1994. - Balkema, 1994. - P. 581-586.

31. Modelling of a Hydrogen Saturated Layer Within the Micropolar Approach / K. Frolova, E. Vilchevskaya, V. Polyanskiy, E. Alekseeva // New Achievements in Continuum Mechanics and Thermodynamics. - Springer, Cham, 2019. - P. 117-128. - DOI: 10.1007/978-3-030-13307-8_9.

32. Adomeit G. Determination of elastic constants of a structured material // Mechanics of Generalized Continua. - 1968. - P. 80-82. - DOI: 10.1007/978-3-662-30257-6_8.

33. Lakes R. S. Size effects and micromechanics of a porous solid // Journal of Materials Science. - 1983. - Vol. 18, no. 9. - P. 2572-2580. - DOI: 10.1007/BF00547573.

34. Yang J. F. C., Lakes R. S. Transient study of couple stress effects in compact bone: torsion // Journal of biomechanical engineering. - 1981. - Vol. 103, no. 4. - P. 275-279.

35. Boundary layer influence on the distribution of hydrogen concentrations during hydrogeninduced cracking test of steels / E. Alekseeva, A. Belyaev, A. Zegzhda, A. Polyanskiy, 
V. Polyanskiy, K. Frolova, Y. Yakovlev // Diagnostics, Resource and Mechanics of materials and structures. - 2018. - Iss. 3. - P. 43-57. - DOI: 10.17804/2410-9908.2018.3.043-057.

36. Surface effect of the waves of plastic deformation and hydrogen distribution in metals / A. K. Belyaev, V. A. Polyanskiy, Y. A. Yakovlev, D. E. Mansyrev, A. M. Polyanskiy // Days on Diffraction : IEEE, 2017. - P. 45-50. - DOI: 10.1109/DD.2017.8167993.

37. Phenomenon of skin effect in metals due to hydrogen absorption / V. A. Polyanskiy, A. K. Belyaev, E. L. Alekseeva, A. M. Polyanskiy, D. A. Tretyakov, Yu. A. Yakovlev // Continuum Mech. Thermodyn. - 2019. - Vol. 31, iss. 6. - P. 1-15. - DOI: 10.1007/s00161-019-00839-2.

38. Martinsson A., Sandstrom R. Hydrogen depth profile in phosphorus-doped, oxygen-free copper after cathodic charging // Journal of Materials Science. - 2012. - Vol. 47, no. 19. - P. 67686776. - DOI: 10.1007/s10853-012-6592-y. 